POS $\quad$ PROCEEDINGS

\title{
Eco-Gas Studies for the CMS iRPC for the High Luminosity LHC
}

\author{
D. Ramírez ${ }^{a}$, S. Carrillo ${ }^{a}$ and M. Ramírez ${ }^{a}$ \\ ${ }^{a}$ Universidad Iberoamericana \\ Physics and Mathematics Department \\ Prol. Paseo de la Reforma 880, Lomas de Santa Fe \\ Mexico City \\ 01219, Mexico \\ E-mail: dalia.lurg@gmail.com, salvador.carrillo@ibero.mx,mateorgf@gmail.com
}

We will present a summary of the performance of the improved RPC (iRPC) using several RPC gas mixtures with a low Global Warming Potential (GWP). We have replaced the Freon (R134a) from the official CMS gas mixture with $\mathrm{HFO}$ and $\mathrm{CO}_{2}$. The results show promising mixtures for the iRPC for the High Luminosity LHC.

7th Annual Conference on Large Hadron Collider Physics - LHCP2019

20-25 May, 2019

Puebla, Mexico 


\section{Global Warming Potential}

The Global Warming Potential (GWP) describes the relative contribution to the greenhouse effect of a gas with respect to $\mathrm{CO}_{2}$ Due to the adoption of the Kyoto Protocol and the Regulations of the European Community, gases with a high impact to the environment (GWP index above 150) are being replaced or banned from gas mixtures. Although the scientific experiments are excluded from this regulations [1], the European Organization for Nuclear Research (CERN) pushes their collaborations to look for replacements of this non eco-friendly gases. In particular, the Compact Muon Solenoid (CMS) experiment looks for an eco-friendly gas mixture to replace the fluor-based gas mixture in their Resistive Plate Detectors (RPCs).

The current gas mixture of CMSs RPC detectors is composed of SF6 0.3\%, Isobutane (iC2H10) $4.5 \%$ and Freon $(\mathrm{C} 2 \mathrm{H} 2 \mathrm{~F} 4)$ in $95.2 \%$, resulting in a GWP index equal to 1433, the main contribution comes from the Freon. In this work, 5 alternative gas mixture with a lower GWP index are studied to look for a similar performance as the aforementioned mixture, besides being friendly to the environment. The $\mathrm{C} 2 \mathrm{H} 2 \mathrm{~F} 2$ is replaced with mixtures of HFO-1234ze ([3]) and $\mathrm{CO} 2$ in different proportions (see table 1). The GWP values of each component and mixture are summarized in table 2 .

Table 1: Gas mixture composition used by CMS RPCs and five more eco-friendly mixtures (freon is replaced by gases with lower Global Warming Potential)

\begin{tabular}{|c|c|c|c|c|c|c|}
\hline Component & CMS & 1 & 2 & 3 & 4 & 5 \\
\hline \hline SF6 & $0.3 \%$ & $0.3 \%$ & $0.6 \%$ & $0.6 \%$ & $0.6 \%$ & $0.6 \%$ \\
\hline Isobutane & $4.5 \%$ & $4.5 \%$ & $4.5 \%$ & $4.5 \%$ & $5 \%$ & $4.5 \%$ \\
\hline $\mathrm{HFO}$ & - & $50 \%$ & $55 \%$ & $50 \%$ & $50 \%$ & $45 \%$ \\
\hline $\mathrm{CO}_{2}$ & - & $45.2 \%$ & $39.9 \%$ & $44.9 \%$ & $44.4 \%$ & $49.9 \%$ \\
\hline Freon & $95.2 \%$ & - & - & - & - & - \\
\hline
\end{tabular}

To get the GWP values of the mixtures, one must multiply the proportion by percentage mass of each component $\left(\mathrm{M} \%{ }_{i}\right)$, times its corresponding GWP value $\left(\mathrm{GWP}_{i}\right)$. Finally the total GWP value of the mixture is the sum of all the values obtained of the corresponding components for each mixture.

$$
\text { GWP of Blend }=\sum_{i=1}^{n} M \%_{i} \times G W P_{i}
$$

\section{Experimental Setup}

An improved RPC (iRPC) detector is used to compare the performance of RPCs detectors using new gas mixtures with respect to the response using the current CMS Mixture. The iRPCs technology, $1.4 \mathrm{~mm}$ gap width, are in development in order to improve the rate capability and the 
Table 2: Global Warming Potential of each gas and mixture used

\begin{tabular}{|c|c||c|c|}
\hline Component & GWP & Mixture & GWP \\
\hline \hline SF6 & 23900 & Mix 1 & 75.14 \\
\hline Isobutane & 3.3 & Mix 2 & 145.6 \\
\hline HFO & 3 & Mix 3 & 145.5 \\
\hline $\mathrm{CO}_{2}$ & 1 & Mix 4 & 145.51 \\
\hline Freon & 1430 & Mix 5 & 146.73 \\
\hline CMS & \multicolumn{3}{|c}{1434.55} \\
\hline \hline
\end{tabular}

aging of RPCs for the next Upgrade of the CMS Detector ([2]).

The iRPC of this experimental setup is a double-gap RPC with dimensions $97 \times 54 \mathrm{~cm}^{2}$ and 32 strips of $2.5 \mathrm{~cm}$ width. A CAEN Module DT5742 was used to digitize 8 analog channels in the detector area covered by a trigger system composed of 3 plastic scintillators (in figure 1 the experimental setup is shown and in figure 2 an example of a digitized signal ). The iRPC detector was operated in a double gap and a single gap mode (just for one gap). Double or single gap mode means the high voltage is applied to both or just one gap.

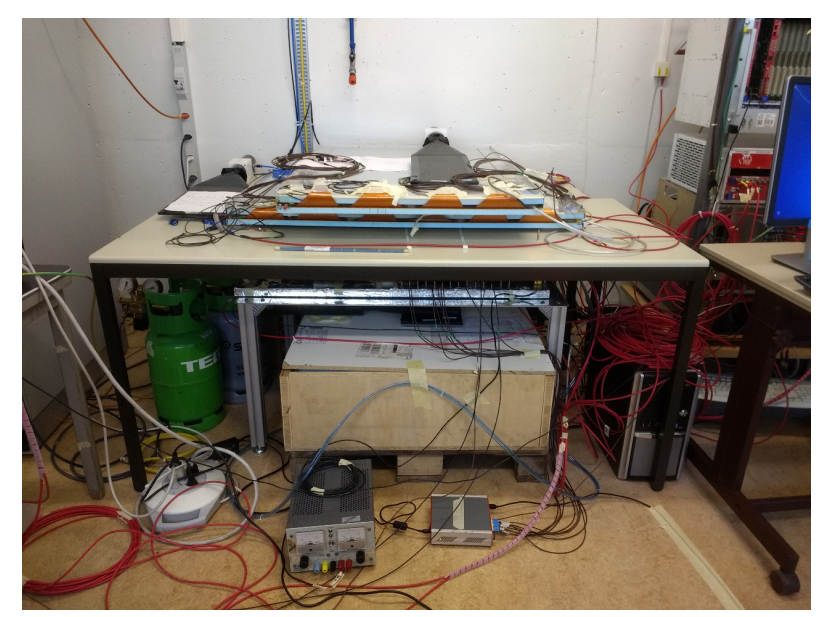

Figure 1: Experimental setup to study the RPC response to different gas mixtures. A 3-scintillators array is used as a trigger for cosmic rays, analog signals from $1.4 \mathrm{~mm}$ gap RPC are digitized using the CAEN Module DT5742 and the RPC is operated in double-gap and single-gap mode. Only one gap was studied in single-gap mode.

\section{Efficiency}

Pulses from the digitized analog channels are analyzed to get the efficiency curve of the iRPC for each gas mixture. A pulse is considered a signal if it overcomes a threshold value defined as 10 times the standard deviation of the 100 first points that compose that pulse. An example of a 
Table 3: Working Point values for the mixtures used, both in double-gap (top row) and single-gap (bottom row) modes.

\begin{tabular}{|c|c|c|c|c|c|c|}
\hline & 1 & 2 & 3 & 4 & 5 & CMS \\
\hline \hline \multirow{2}{*}{ WP \pm error $[\mathrm{V}]$} & $8451.9 \pm 13.4$ & $8870.5 \pm 7.4$ & $8529.3 \pm 6.8$ & $8563.5 \pm 7.4$ & $8255.6 \pm 7.4$ & $7185.8 \pm 4.5$ \\
\cline { 2 - 7 } & - & $9129.3 \pm 14$ & $8736.6 \pm 13.9$ & $8815.6 \pm 14.5$ & $8545 \pm 25.7$ & $7467.2 \pm 6.02$ \\
\hline
\end{tabular}

typical analog signal obtained with the iRPC using a new gas mixture and the current CMS Mixture is shown in (figure 2).

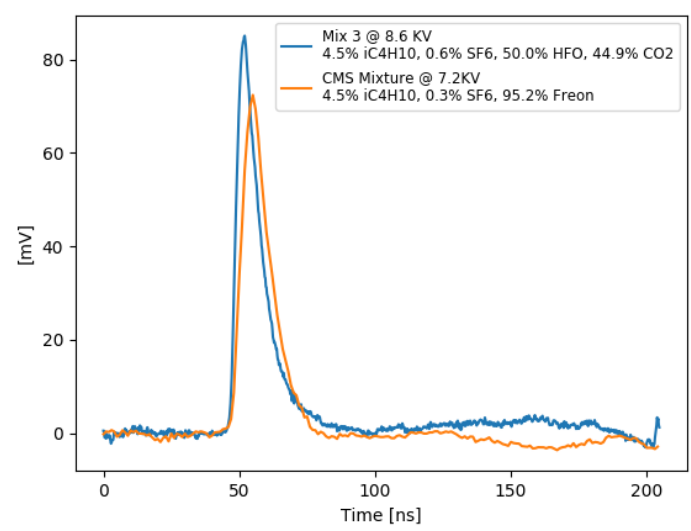

Figure 2: Example of a typical analog signal obtained with an RPC using a gas mixture of 50\% HFO, $44.9 \%$ $\mathrm{CO} 2,0.6 \% \mathrm{SF} 6$ and $4.5 \% \mathrm{iC} 4 \mathrm{H} 10$ at $8.6 \mathrm{KV}$, compared with an analog signal using the CMS RPC mixture $0.3 \%$ SF6, $4.5 \%$ iC4H10 and $95.2 \%$ Freon.

The efficiency was calculated as the number of events with signal in at least one strip, divided by the total number of events. This was done at several HV points for all mixtures at table 1 in double-gap mode and in single-gap mode (except for Mixture 1). The high voltage working point as the voltage when the efficiency reaches the $95 \%$ plus $150 \mathrm{~V}\left(W P=H V_{95 \% E f f}+150 \mathrm{~V}\right)$. Table 3 summarizes the working point values of each mixture in double-gap and single-gap modes.

Figure 3 and figure 4 shows the efficiency based on the effective high voltage for double-gap and single-gap modes, respectively. In general a similar behavior between all mixtures is observed and, although the efficiency curve is shifted to higher HV values, the WP is still acceptable. 


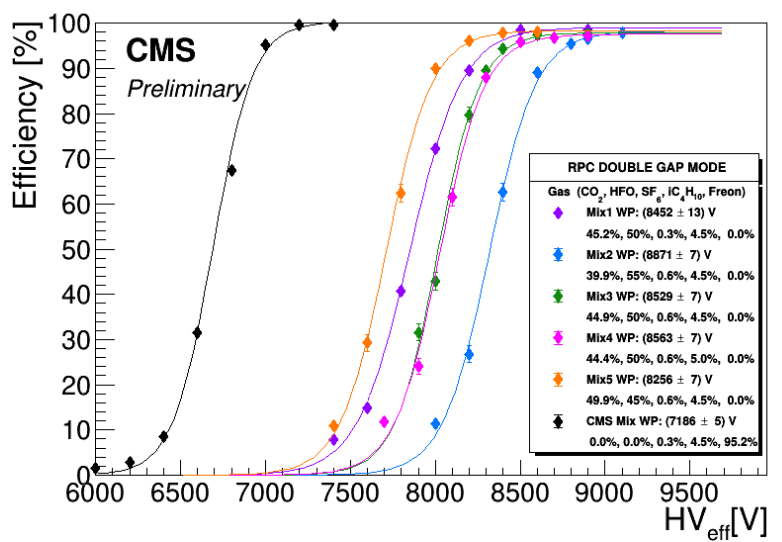

Figure 3: Efficiency curves and HV Working Point for several gas mixtures obtained with the RPC operating in double-gap mode. Working Point is defined as $\mathrm{HV}_{95 \% E f f}+150 \mathrm{~V}$.

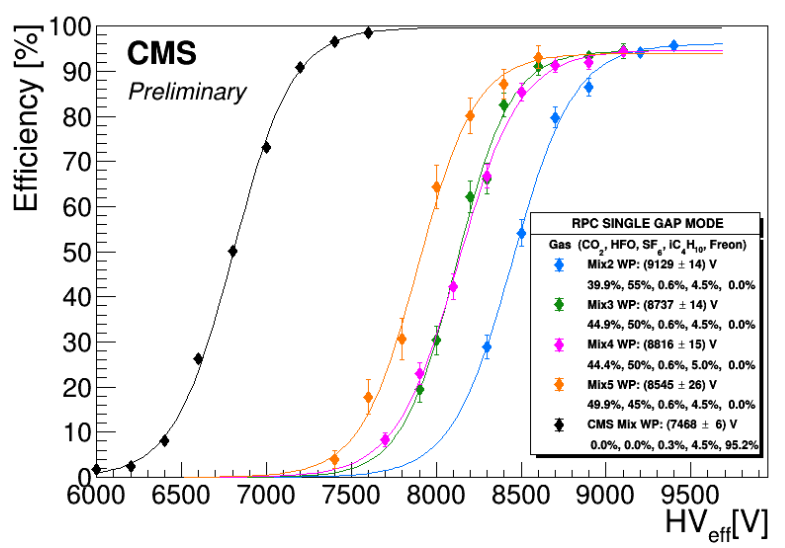

Figure 4: Efficiency curves and HV Working Point for several gas mixtures obtained with the RPC operating in double-gap mode. Working Point is defined as $\mathrm{HV}_{95 \% E f f}+150 \mathrm{~V}$.

With these efficiency curves we can compare some of the mixtures like those shown in figure 5 and figure 6, where we see mixtures 3 and 4 for both double and single gaps. In both cases, a similar behavior is observed for both mixtures. The isobutane $0.5 \%$ of the mixture 3 to 4 was increased and a decrease of $\mathrm{CO}_{2}$ of $0.5 \%$ of the mixture 3 to 4 .

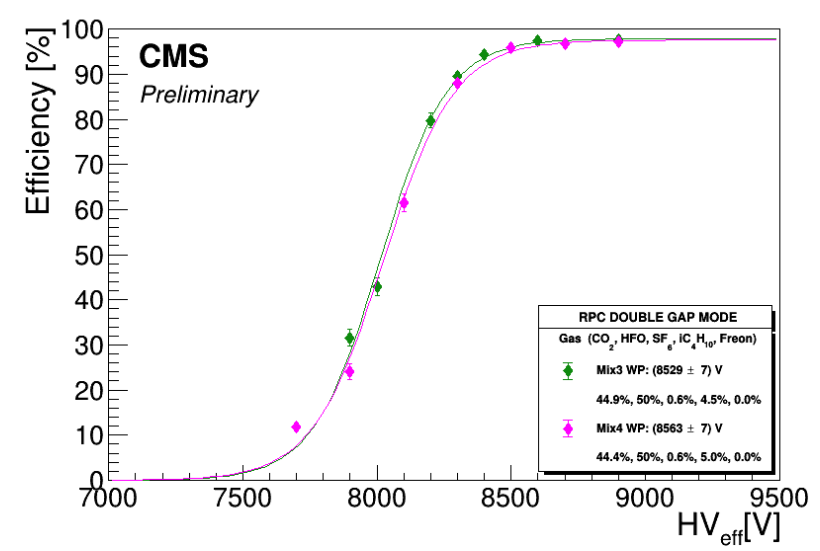


Figure 5: Efficiency curves and HV Working Point for mixtures 3 and 4 obtained with the RPC operating in double-gap mode. From 3 to 4 mixtures, isobutane is increased $0.5 \%$ decreasing $\mathrm{CO}_{2}$. A similar behavior is observed.

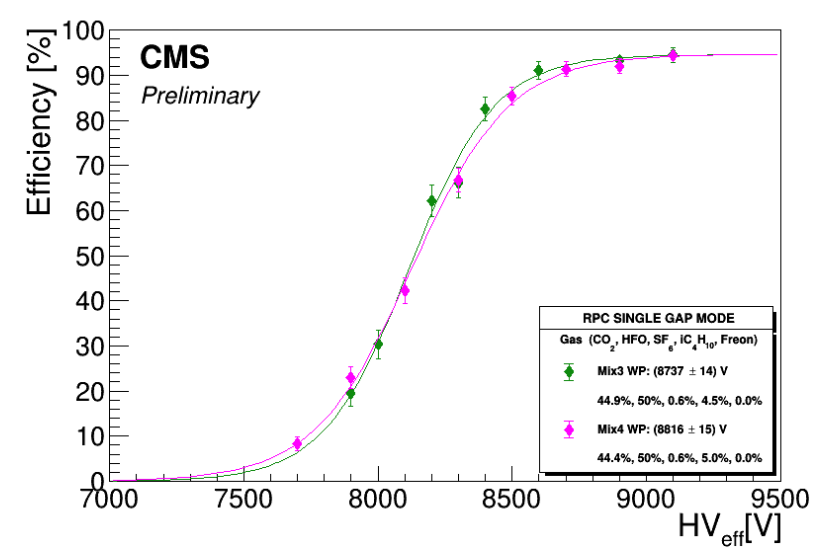

Figure 6: Efficiency curves and HV Working Point for mixtures 3 and 4 obtained with the RPC operating in single-gap mode. From 3 to 4 mixtures, isobutane is increased $0.5 \%$ decreasing $\mathrm{CO}_{2}$. A similar behavior is observed.

On the other hand, in figure 7 and figure 8 the mixes 2, 3 and 5 are shown, from which we can conclude that if the HFO is increased by $5 \%$ (from mixture 5 to 3 and from 3 to 2) we will obtain an increase in the HV WP (High Voltage Working Point defined as WP $=H V_{95 \% \text { fficiency }}+150 \mathrm{~V}$ ) of around $300 \mathrm{~V}$.

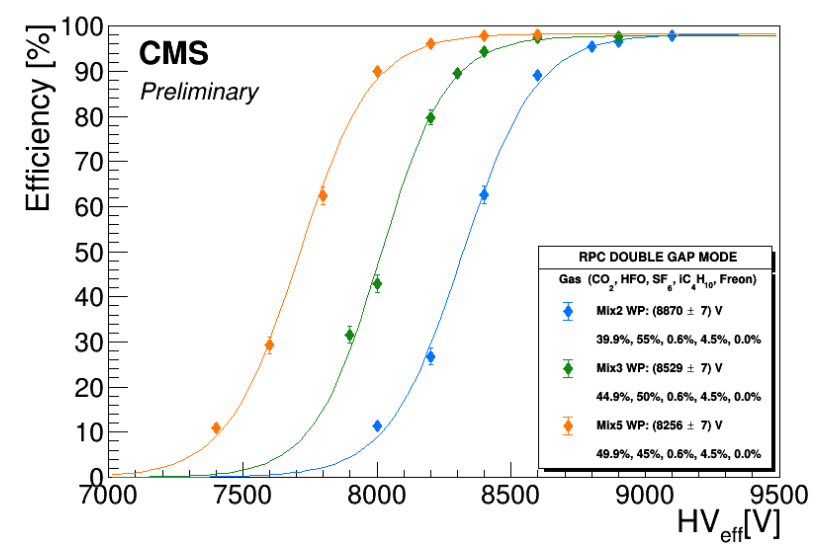

Figure 7: Efficiency curves and HV Working Point for mixtures 2, 3 and 5 obtained with the RPC operating in double-gap mode. From left to right efficiency curves, HFO is increased $5 \%$ decreasing $\mathrm{CO}_{2}$. The Working Point increased $\sim 300 \mathrm{~V}$. 


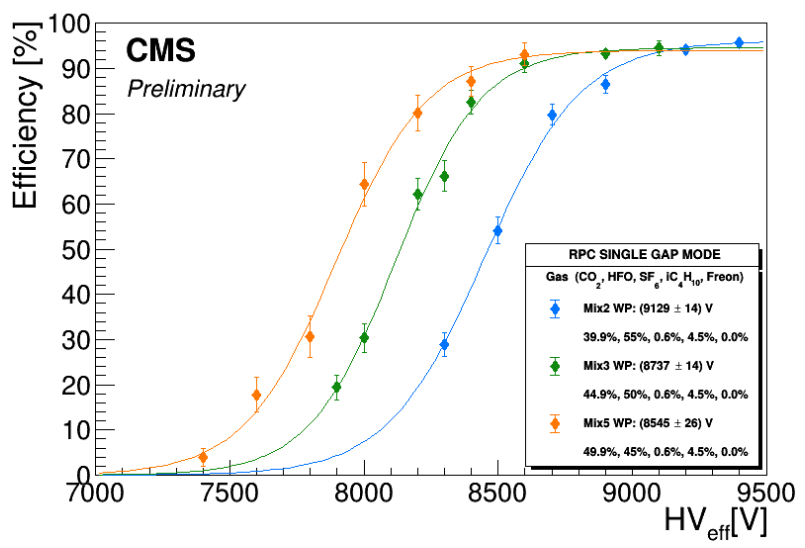

Figure 8: Efficiency curves and HV Working Point for mixtures 2, 3 and 5 obtained with the RPC operating in single-gap mode. From left to right efficiency curves, HFO is increased 5\% decreasing $\mathrm{CO}_{2}$. The Working Point increased $\sim 300 \mathrm{~V}$. 


\section{Cluster Size}

Cluster size was defined as the number of consecutive strips with signal above the threshold. Figure 9 and 10 show the cluster size versus the Working Point for double-gap and single-gap modes, the latter considering both the top and the bottom for the CMS mixture.

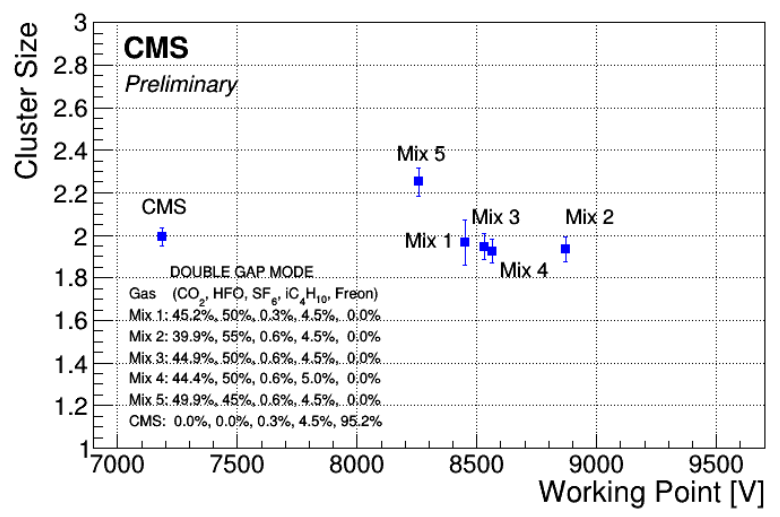

Figure 9: Cluster Size versus the Working Point with the RPC operating in double-gap mode shows an almost constant behavior for mixtures 1 to 4 and compatible with the CMS mixture value, mixture 5 is considered far away.

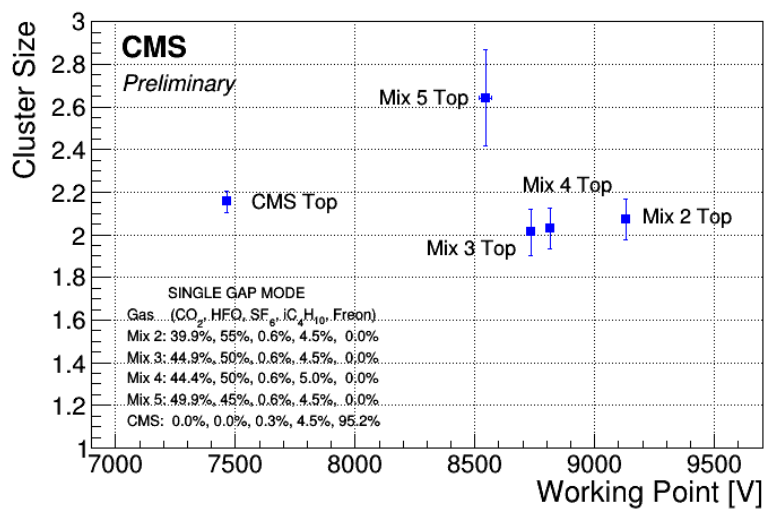

Figure 10: Cluster Size versus the Working Point with the RPC operating in single-gap mode shows an almost constant behavior for all mixtures. 


\section{Probability of cluster greater than 6}

Here we consider the probability of cluster size with more than 6 consecutive strips. In figures 11 and 12 this probability is shown for double-gap and single-gap modes, respectively.

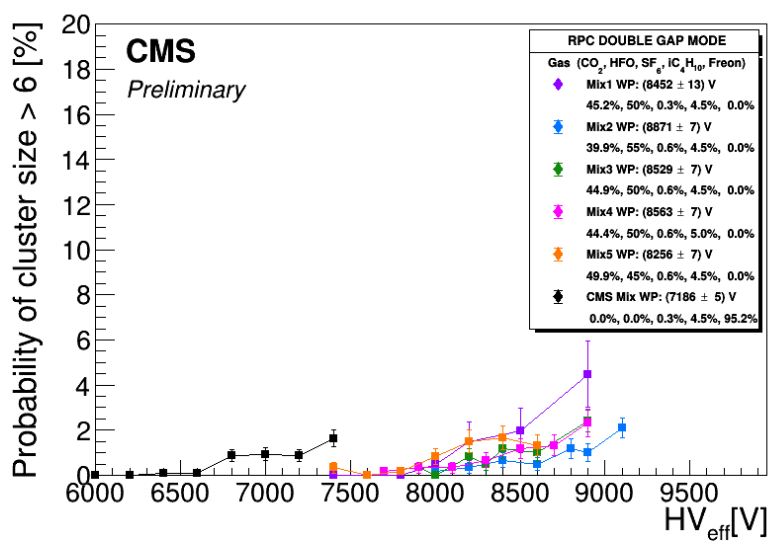

Figure 11: Probability of event with cluster size greater than 6 for different gas mixtures obtained with the RPC operating in double-gap mode. Shows a good RPC response at the Working Point and close to the CMS mixture value.

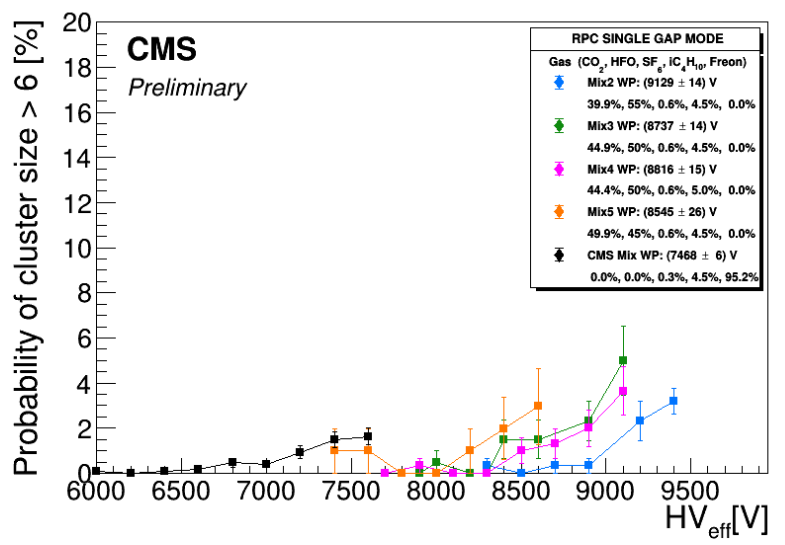

Figure 12: Probability of event with cluster size greater than 6 for different gas mixtures obtained with the RPC operating in single-gap mode. Shows a good RPC response at the Working Point and close to the CMS mixture value. 


\section{Conclusion}

With this first study results were obtained on some promising mixtures to replace the current mix of these detectors, the most promising mixtures are 3 and 4 due to its behavior showed in this work. However it is necessary to study them in depth in order to conclude if any of them could truly replace the CMS mixture for the High Luminosity LHC.

However, the proposed mixtures satisfy the condition of having a low GWP index, and their performance, allows us to say, those are good alternatives to replace the actual gas mixture in the CMS RPC detectors.

\section{Credits}

We are deeply grateful with the Gas-Group EP-DT: R. Guida, B. Mandelli, the "Universidad Iberoamericana", CONACyT, the "Sociedad Mexicana de Física", the "División de Partículas y Campos" and to CMS-RPC group for their support and feedback.

\section{References}

[1] M.Abbrescia et al., Resistive Gaseous Detectors: Design, Performance and Perspectives, Wiley-VCH, Bari, 2018.

[2] CMS Collaboration, The Phase-2 Upgrade of the CMS Muon Detectors, Technical Design Report, 2017.

[3] J. Steven Brown, HFOs New Low Global Warming Potential Refrigerants, 2009. 